\title{
Investigation into the Cause of Spontaneous Emulsification of a Free Steel Droplet; Validation of the Chemical Exchange Pathway
}

\author{
STEPHEN SPOONER, ANDRE N. ASSIS, JASON WARNETT, RICHARD FRUEHAN, \\ MARK A. WILLIAMS, and SEETHARAMAN SRIDHAR
}

\begin{abstract}
Small Fe-based droplets have been heated to a molten phase suspended within a slag medium to replicate a partial environment within the basic oxygen furnace (BOF). The confocal scanning laser microscope (CSLM) has been used as a heating platform to interrogate the effect of impurities and their transfer across the metal/slag interface, on the emulsification of the droplet into the slag medium. The samples were then examined through X-ray computer tomography (XCT) giving the mapping of emulsion dispersion in 3D space, calculating the changing of interfacial area between the two materials, and changes of material volume due to material transfer between metal and slag. Null experiments to rule out thermal gradients being the cause of emulsification have been conducted as well as replication of the previously reported study by Assis et al $^{[1]}$ which has given insights into the mechanism of emulsification. Finally chemical analysis was conducted to discover the transfer of oxygen to be the cause of emulsification, leading to a new study of a system with undergoing oxygen equilibration.
\end{abstract}

DOI: $10.1007 / \mathrm{s} 11663-016-0700-3$

(C) The Author(s) 2016. This article is published with open access at Springerlink.com

\section{INTRODUCTION}

MANY steps in the process of steel making rely on the transfer of elements from metal to slag; a few examples are silica removal in the blast furnace, ${ }^{[2]}$ sulfur in the desulfurization pre-treatment,${ }^{[3]}$ and phosphorus removal during steelmaking in the basic oxygen furnace (BOF) ${ }^{[4]}$ In the case of phosphorus refining in the BOF, much work has been carried out on the thermodynamic equilibrium between the metal and slag, ${ }^{[5-7]}$ giving evidence to the kinetic inhibition of the potential partition possible. ${ }^{[8]}$ This lack of equilibrium could either be caused by lack of homogeneity in the slag layer of the BOF, (reducing the local driving force for phosphorus partition into the slag), or through kinetic restrictions through a deficiency of required interface for refining to transpose across/mass transfer controlling delivery of impurities to the interface. ${ }^{[8-10]}$ Due to the inherent high temperatures, the transfer of material at the interface tends to be fast in comparison to the kinetically restricting mass transfer of materials in their respective bulk phases. ${ }^{[8-10]}$ Current levels of understanding have extended beyond equilibrium conditions

STEPHEN SPOONER, Ph.D. Researcher, JASON WARNETT, Research Fellow, and MARK A. WILLIAMS and SEETHARAMAN SRIDHAR, Professors, are with the University of Warwick, Coventry, UK. Contact e-mail: s.r.a.spooner@warwick.ac.uk ANDRE N. ASSIS, formerly Researcher with Carnegie Mellon University, Pittsburgh, PA, is now R\&D Project Manager with Vallourec Research Center, Aulnoye-Aymeries, France. RICHARD FRUEHAN, Professor, is with Carnegie Mellon University.

Manuscript submitted January 21, 2016

Article published online May 31, 2016. to include the description of mass transport in the slag and metal phases. ${ }^{[1-14]}$ It is however known that under dynamic conditions the interface between phases can display significant perturbation in comparison to a relaxed planar state, and may even lead to emulsification. ${ }^{[15-20]}$ A proper description of the slag/metal reaction necessitates an elucidation of the dynamic change of interfacial area and the coupling of this change to the interfacial reactions. As such this work intends to investigate the effects of refining performance on interface morphology between $\mathrm{Fe}$ alloys and slags where mixing of phases may intrinsically occur to reduce the kinetic restrictions of mass transfer. Understanding when and by how much areas change during a reaction would be critical when describing the transient trajectory towards equilibrium in metallurgical processes.

In this study the specific case of the transient interface in the BOF emulsion phase is considered, which has previously been attributed to offering large contributions to the overall refining performance seen within the BOF $^{[21]}$ The emulsion phase of the BOF is a mix of slag, gas $\left(\mathrm{O}_{2}, \mathrm{CO}, \mathrm{CO}_{2}\right)$, and metal droplets caused by the impinging oxygen jet delivered to the bulk bath surface overcoming the surface tension of the molten metal and sheering material away to form discrete droplets (as well as larger plumes of metal). ${ }^{[22,23]}$

The interface of metal and slag in this emulsion phase consists of several factors:

1. Amount of metal in the emulsion

2. How long the metal stays in the emulsion (average residence time)

3. The size of discrete metal droplets

4. The morphology of metal droplets 
Table I. A Summary of the Previous Findings of Metal Droplet Size in Oxygen Steelmaking Emulsions

\begin{tabular}{|c|c|c|}
\hline Researchers & Place of Collection & Droplet Size Range $(\mu \mathrm{m})$ \\
\hline $\mathrm{IMPHOS}^{[45]}$ & inside pilot converter, special lance & 16 to 6360 \\
\hline $\operatorname{Resch}^{[46]}$ & paused and tilted converter & 50 to 2000 \\
\hline Tokovoi et al. ${ }^{[47]}$ & upper slag/metal emulsion & 1000 to 2500 \\
\hline Cicutti ${ }^{[21]}$ & inside full converter, special lance & 230 to 3350 \\
\hline Koria et al. ${ }^{[48]}$ & slashed liquid outside crucible & 40 to 70,000 \\
\hline Baptizmanskii et al. ${ }^{[49]}$ & cutting hole in crucible wall & 50 to 18000 \\
\hline Meyer ${ }^{[50]}$ & through tap hole, outside converter & 150 to 3320 \\
\hline Block et al. ${ }^{[51]}$ & 50 to $150 \mathrm{~mm}$ above bulk bath inside converter & 500 to 4000 \\
\hline Urquhart et al..$^{[52]}$ & inside full converter, special lance & 63 to 2000 \\
\hline
\end{tabular}

Previous studies, including that of the present authors have investigated the macroscopic elements (points $1 \&$ 2 above) of this interface. Reported residence times ranging from 0.4 to 120 seconds are given, with an average of around 40 seconds. ${ }^{[24-30]}$ The amount of metal in the emulsion has been reported in a complete range of up to 50 pct of the tap weight dependant on blow time. ${ }^{[31,32]}$

With regard to the kinetics of a specific metal droplet in the emulsion, several authors have investigated variable aspects (point $3 \& 4$ above). Studies have been carried out both on industrial scale and laboratory scale on the size of quenched static samples; these results as well as how they were obtained are shown in Table I.

More recently work began into the tracking of a droplet transient morphology during refining processes. An example in this area is the work conducted by Rhamdhani et al. ${ }^{[18]}$ where $\mathrm{Fe}-\mathrm{Al}$ droplets were immersed in $\mathrm{CaO}-\mathrm{SiO}_{2}-\mathrm{Al}_{2} \mathrm{O}_{3}$, the recovered metal droplets were then measured using a system of paper standards covering the droplet surface, giving an effective but rudimental method of calculating the change in surface area. X-ray fluoroscopy and direct observation of in situ droplets has also given evidence to the change in droplet shape during Fe alloy/slag interactions. ${ }^{[16,33-35]}$ It has been proposed by these studies that the initial driving force is integral when evaluating the change in droplet morphology as the interfacial tension between the two mediums decreases rapidly allowing for interfacial area increase.

The use of microscopy is perhaps the most essential tool for investigative material science offering the ability to discern micro-structures at finer and finer scales; but usually a posteriori to a process. Professor Toshihiko Emi pioneered the use of laser confocal optics with an Au-image furnace allowing for in situ imaging, and his group at Tohoku University was the first to document the clustering of inclusions, ${ }^{[36,37]}$ peritectic solidification, ${ }^{[38]}$ and particle pushing. ${ }^{[39]}$

The present work, inspired by Prof. Emi's stellar publications, leads on from the initial findings of Assis et al. ${ }^{[1]}$ where high-temperature confocal scanning laser microscopy (HT-CSLM) and micro X-ray computer tomography (XCT) were coupled together to provide insight into the full transient morphology of an Fe-P 0.2 wt pet alloy droplet submerged in slag similar to that seen in end blow BOF refining. HT-CSLM was used to provide a high heating rate reaction platform and XCT was used to offer 5 micron resolution 3D quantification of the metal-slag interface of quenched samples.

Initially a repeat of the previously reported work has been carried out to offer consideration to the consistency of the intricate technique used to produce the samples required due to the significant differences with $\mathrm{Rhamd-}$ hani's ${ }^{[18-20]}$ findings and give insights into the mechanism of spontaneous emulsification. This has been followed by initial chemical analysis of the samples from the previous study. ${ }^{[1]}$ The results of which indicate the need for a further trial, where no phosphorus was present, leading to the exploration of oxygen transition across the interface causing spontaneous emulsification. A system at near equilibrium has also been studied to interrogate the need for a chemical exchange to cause the emulsification. The objectives of this work are to investigate (i) whether emulsification observed in the experimental drops are indeed caused by a chemical exchange (by comparing with a non-reactive system) and not by the experimental technique and (ii) to attempt to isolate whether it is caused by the transfer of phosphorous, transfer of oxygen, or both.

\section{EXPERIMENTAL}

The experimental setup consists of a HT-CSLM, used for its high heating and cooling rates. This is followed by XCT to give $3 \mathrm{D}$ representations of an entire sample. XCT also allows for identification of a representative slice through a sample that can then be sectioned, polished, and chemical analysis performed. The chemical analysis techniques used are: Inductively coupled plasma-mass spectrometry (ICP-MS), wavelength dispersive spectroscopy-scanning electron microscope (WDS-SEM), laser ablation-inductively coupled plasma mass spectrometry (LA-ICPMS), and secondary ion mass spectrometry (SIMS).

\section{A. Materials \& Methods}

The material used in the initial study has been reported previously. ${ }^{[1]}$ In summary, it consists of a Fe-0.2 wt pet $\mathrm{P}$ alloy, which was reacted with a slag 
Table II. ICP Detected Chemical Composition of Fe alloys Used in this Study

\begin{tabular}{lllllllllr}
\hline & Pct Mn & Pct P & Pct Ni & Pct Cr & Pct Al & Pct C & Pct S & Pct O & Pct N \\
\hline a1 & 0.011 & 0.201 & 0.003 & 0.002 & 0.002 & 0.0008 & 0.0012 & 0.0737 & 0.0005 \\
a2 & 0.0003 & 0.0004 & 0.0001 & 0.0003 & 0.0005 & 0.004 & 0.001 & 0.0034 & $<0.001$ \\
\hline
\end{tabular}

Table III. XRF Detected Chemical Composition of Master Slags, Used in this Study and by Assis et al.

\begin{tabular}{lccccccc}
\hline & $\mathrm{Pct} \mathrm{CaO}$ & $\mathrm{Pct} \mathrm{MgO}$ & $\mathrm{Pct} \mathrm{SiO}$ & $\mathrm{Pct} \mathrm{FeO}_{\mathrm{t}}$ & $\mathrm{Pct}_{2} \mathrm{O}_{5}$ & $\mathrm{CaO}_{2} \mathrm{SiO}_{2}$ & $\mathrm{Pct}_{2} \mathrm{Al}_{3}$ \\
\hline $\mathrm{S} 1$ & 36.64 & 7.08 & 16.98 & 33.56 & 1.66 & 2.16 & 0 \\
$\mathrm{~S} 2$ & 36.89 & 7.14 & 16.21 & 32.31 & 1.65 & 2.28 & 0 \\
$\mathrm{~S} 3$ & 38.15 & 10.26 & 16.89 & 34.70 & 0.01 & 2.26 & 0 \\
$\mathrm{~S} 4$ & 43.02 & 8.74 & 24.11 & 0 & 0 & 1.78 & 24.13 \\
\hline
\end{tabular}

Table IV. Heating and Quenching Regime of All Samples

\begin{tabular}{lllll}
\hline & Step 1 & \multicolumn{1}{c}{ Step 2 } & \multicolumn{1}{c}{ Step 3 } & Step 4 \\
\hline Temp (K) & Rt-373.2 & $373.2->1873.2$ & S1873.2 $->773.2$ & $773.2->$ Rt \\
Heating Rate $\left(\mathrm{K} \mathrm{min}^{-1}\right)$ & 50 & 500 & -1200 & -300 \\
Hold Time (s) & 45 & $\# \#$ & 70 & - \\
\hline
\end{tabular}

\#\# being the period of time a sample was held at high temperature for.

whose X-ray fluoroscopy (XRF) chemical analysis can be seen in Table III, this will be called S1.

A repeat of the system described by Assis et al..${ }^{[1]}$ has been conducted. An Fe- 0.2 wt pct alloy droplet of $17 \mathrm{mg}$ $\pm 0.4 \mathrm{mg}$ with a cylindrical input geometry of $1.19 \mathrm{~mm}$ $\mathrm{H} 1.49 \mathrm{~mm} \mathrm{D}$ is used (the alloy has the exact same composition as used in the initial study, seen in Table II. This will be called alloyl (a1). A master slag of the composition seen in Table III (analysis via XRF) was prepared under shield gas argon in an induction furnace at $1873 \mathrm{~K}\left(1600{ }^{\circ} \mathrm{C}\right)$ from the stock materials, $\mathrm{CaO}$, $\mathrm{SiO}_{2}, \mathrm{MgO}, \mathrm{Fe}_{2} \mathrm{O}_{3}$, and $\mathrm{Ca}_{2} \mathrm{P}_{2} \mathrm{O}_{7}$; this will be called $\mathrm{S} 2$.

The second set of material used consists of a $2-\mathrm{mm}$ diameter iron wire supplied by Alfa Aesar, cut into disks using a low speed diamond blade saw. This will be called alloy 2 (a2), the composition of which can be seen in Table II. The weight of the resulting disks was between 14 and $16 \mathrm{mg}$. A master slag for this was prepared with the same reagent grade powders as previously stated inside an $\mathrm{MgO}$ crucible, melted for $1873 \mathrm{~K}\left(1600^{\circ} \mathrm{C}\right)$ for 2 hours in a tube furnace under high purity argon to ensure homogeneous mixing. The crucible was then grinded, slag pulverized, and analyzed via XRF (the composition can be seen in Table III). This will be called S3. A further slag composition was created in the same way to be in near equilibrium with a2 with respect to oxygen partition; this slag will be referred to as S4 and the composition is seen in Table III.

\section{B. High-Temperature Confocal Scanning Laser Microscope}

$\mathrm{MgO}$ crucibles $(9 \mathrm{~mm}$ inner diameter and $4.5 \mathrm{~mm}$ height) were packed with $0.45 \mathrm{~g} \pm 0.06 \mathrm{~g}$ of the respective master slag. The crucible was then loaded into the HT-CSLM, and the chamber was cycled 3 times for ten minutes each through vacuum and argon backfilling (99.9999 pet argon). The slag was pre-melted at $1898 \mathrm{~K}$ $\left(1625^{\circ} \mathrm{C}\right)$ for 30 seconds and then cooled at a nominal rate of $1000 \mathrm{~K} \mathrm{~min}^{-1}$ with helium gas. The crucible is then removed from the chamber and a droplet is loaded into the center of the slag meniscus surface. A further $0.15 \pm 0.042 \mathrm{~g}$ of slag powder is hand pressed on top to ensure the droplet gets submerged when in liquid state. The entire sample was reloaded, cycled 3 times again, and the experiment is then run following the regime shown in Table IV, under $200 \mathrm{ml} \mathrm{m^{-1 }}$ argon flow. Steps 1 and 4 are required to reduce the chance of damaging the bulb.

This was the sample for all systems apart from that of $\mathrm{S} 4$, where a sapphire crucible was used in order to increase scattered light detection, giving a way to effectively increase the depth of focus field for the UV laser.

\section{X-ray Computer Tomography}

Samples containing S1 and S3, were scanned using a 'Nikon Metrology XT H 320 LC' XCT scanner and samples containing S2 and S4 were scanned using a 'Zeiss Versa 520.' The difference in machines was due to the availability during experiments, with the Zeiss preferred due to its better signal-to-noise ratio within images. This arises from different source and detectors within the systems, and a smaller source/detector distance in the Zeiss machine that is better suited for higher-resolution tomography 
Table V. Parameters Used Within the Two Scanners

\begin{tabular}{lll}
\hline & Nikon & Zeiss \\
\hline Voltage $(\mathrm{kV}$ & 220 & 140 \\
Power $(\mathrm{W})$ & 3.1 & 10 \\
Exposure & 4 & 3 \\
Projections & 3142 & 3142 \\
Filter (mm) & $\mathrm{Cu} 0.5$ & $\mathrm{CaF}_{2} 1.0$ \\
Magnification & $\times 39.5$ & $\times 6.69^{*}$ \\
Voxel size & 5.06 & 4.90 \\
\hline
\end{tabular}

* There is an additional focusing step performed by an optic in the Zeiss machine, where $\times 0.4$ was used in this study.

The Nikon machine consists of a $225 \mathrm{kV}$ micro focus with tungsten reflection target and a flat panel detector consisting of $2000 \times 2000$ pixels, each of 200 microns in size. The Zeiss machine has a $160 \mathrm{kV}$ micro focus source with an alumina transmission target and charge-couple device-based detector $2000 \times 2000$ pixels, with a size dependant on the optic used $(\times 0.4$ optic was used in this study with 1.75 micron pixel size).

The parameters for each scan are shown in Table V. The scans were then reconstructed in proprietary packages included within the systems that use filtered back projection. The reconstructed $3 \mathrm{D}$ volume was then evaluated in 'VGStudio MAX' (Volume Graphics $\mathrm{GmbH}$, Germany). Further information on the XCT scanning including reference pieces used for scaling, determination of the droplet isosurface, and validation of the scanning parameters can be found in previous works. ${ }^{[1,40]}$

\section{Chemical Analysis}

In order to evaluate the phosphorus content in the metal before and during emulsification for system 1 samples, WDS-SEM, LA-ICPMS, and SIMS were used. Each of these techniques has the advantage of having small spot sizes which make it possible to chemically analyze the metal drop and emulsion.

WDS-SEM was the initially selected tool to analyze the phosphorus content in the metal drops as it is a non-destructive technique and relatively simple to use. WDS offers better peak resolution and separation than EDS and can potentially detect concentrations below 1 wt pct with the usage of proper standards. Unlike EDS, the detector reads and counts X-rays generated of a single wavelength at a time that are diffracted by a crystal of proper lattice spacing. In the present study the standards used were pure $\mathrm{Fe}$ and $\mathrm{GaP}$ for iron and phosphorus, respectively. Each of the standards were used to standardize accelerating beam voltages of 25 and $30 \mathrm{kV}$ with spot sizes of 6 and 7 microns. The high accelerating voltages and bigger spot sizes were necessary in order to achieve the highest beam current possible in the SEM, which helps in quantifying low concentrations.

LA-ICPMS differs from regular ICP-MS in that it uses a focused laser beam to ablate a small area of the sample. This technique was used to confirm the data acquired by WDS and only one sample was analyzed via this technique. A total of four measurements were taken using ICP-MS across different regions of the metal drop. Each "shot" ablated roughly $5 \mu \mathrm{m}$ in depth with a spot size of $100 \mu \mathrm{m}$ in diameter.

SIMS was used for the analysis of oxygen in the 20 -second sample. SIMS uses a focused ion beam to sputter the surface of the sample. As the ion beam mills the surface, secondary ions are generated and these ions are analyzed by a mass spectrometer. Quantification was based on a stainless steel 302 standard.

\section{RESULTS AND DISCUSSION}

The interaction of metal droplets with slag has been interrogated via the CT scanning of quenched samples produced in the HT-CSLM. The first series reported by Assis et al. ${ }^{[1]}$ has now undergone initial chemical analysis of representative sectioned surfaces (a1 and S1). Secondly a repeat of these initial conditions has been completed to interrogate the consistency of the previously reported tomography results (a1 and S2), as well as further samples taken in the period before emulsification to give insight into the pathway of the droplet breakup. Thus these experiments are very similar and both will be referred to as system 1. As a result of the initial chemical analysis of system 1, a low phosphorus system has been created and analyzed to investigate the true cause of emulsification (a2 and S3, system 2) in the previous works. Finally a system at near equilibrium has been inspected via in situ imaging in the CSLM (a2 and S4, system 3).

\section{A. The Pathways of Emulsification}

XCT scans of quenched samples for a system (system 1) have been reconstructed to produce images seen in Figure 1. It can be seen from Figure 1 that emulsification of the droplet in S2 has occurred.

Several points should be taken from these initial images that display significant differences from the original study. Firstly, the holding times of these samples cross over, and extend beyond that of the originals ( 0 to 120 seconds). This is most likely because the repeat samples were run on a different HT-CSLM by a different user; this resulted in a diverted regime, where it was found that the droplet was taking longer to melt than seen before. At 90 seconds the sample is molten with a spherical geometry and the surface can be seen to have begun to roughen, either through the starting of initial emulsification, or due to the shrinkage of the droplet in an uneven manner during quenching.

The next observation is the pathway of emulsification to coalescence is seen to differ significantly from previously reported. This is thought to be due to the slower heating rates seen within the new samples. A slower heating rate will have caused slag and metal to become molten at a greater time separation. This will potentially reduce the initial driving force of partition equilibration, as material will begin to exchange before the droplet is fully molten. Due to this slower heating rate and the need to hold material at high temperature 

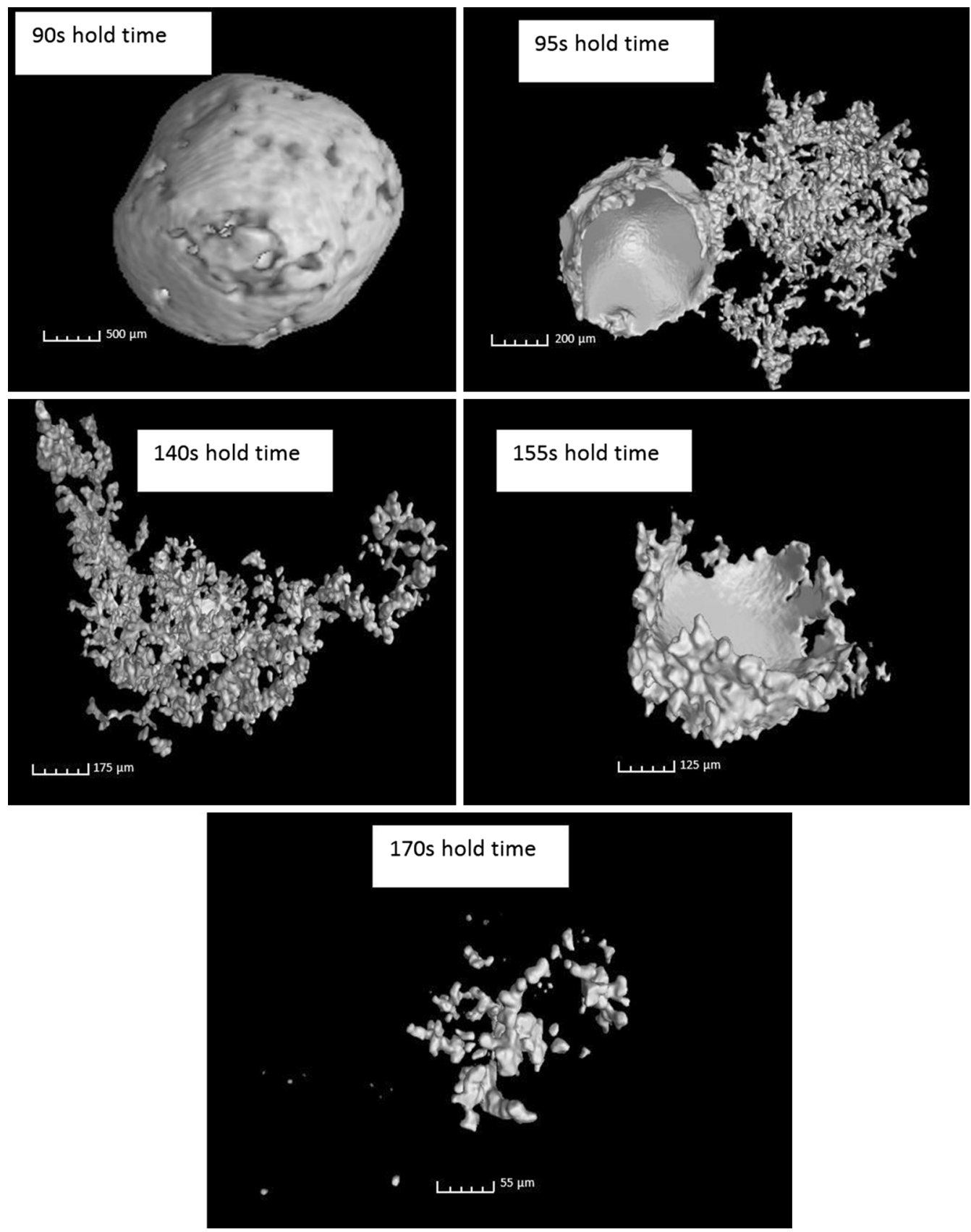

Fig. 1-Reconstructed images from XCT scanning, showing only the metal fraction of the sample.

for longer periods of time, the amount of droplet dissolved into the slag could greatly increase. There are two proposed pathways for the new samples in system 1:

1. The droplet reaches molten state slowly, with initial refining taking place ( 90 seconds), emulsification begins with half the droplet breaking away (95 seconds), the remaining sample continues to emulsify (140 seconds), and then coalesces (155 seconds). Finally the sample continues to be dissolved into the slag, reducing the visible material (170 seconds).

2. Up to 90 seconds the same behavior, full emulsification where some of the sample pools back together due to gravitational effects through porous areas (95 seconds), emulsification continues through to 155 seconds (with pooling due to porosity in the slag happening in 110- and 155 -second samples). Finally after 170 seconds large amounts of the sample have either dissolved or dispersed below XCT resolution.

The pooling theory is due to the orientation of the half-domed sections of the sample, where the cup faces upwards, and has large areas of porosity seen above (see Figure 2). Any emulsified material here would have dropped (have no support to be separated) under quenching conditions.

Finally it can be observed that a large amount of material is being lost throughout the reaction (briefly 


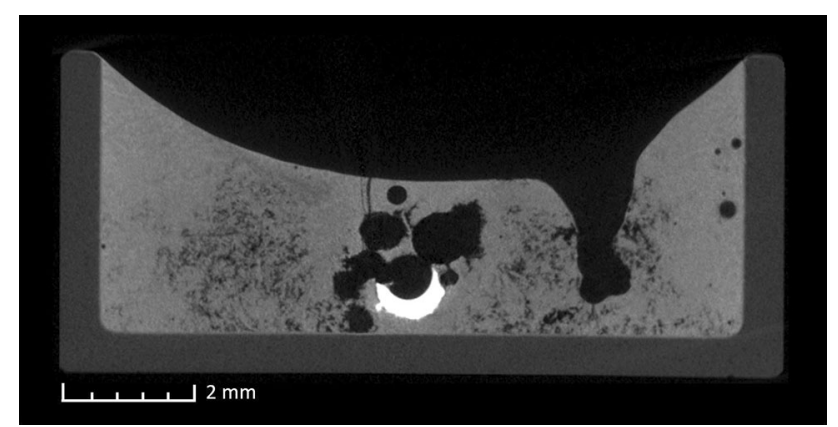

Fig. 2-2D slice of 95-s sample showing the orientation of the domed part of the sample.

mentioned in the previous point). At this stage, the two options are either emulsification beyond the resolution of the current CT scans, or dissolving of the Fe-P droplet into the slag medium. It is believed that the 155 -second sample is coalesced, and due to the relatively high holding time of 170 seconds significant amounts of dissolution is the most plausible result. In the future higher-resolution XCT scanning and SEM imaging will be used to investigate the presence of smaller particles. XRF analysis of the slag may also be carried out to investigate if the Fe content has increased.

\section{B. Chemical Analysis of System 1}

The expected reaction in system 1 is:

$$
5(\mathrm{FeO})+2[\mathrm{P}] \rightarrow\left(\mathrm{P}_{2} \mathrm{O}_{5}\right)+5[\mathrm{Fe}],
$$

where the material in () is in the slag, and those in [] are in the metal droplet.

As previously mentioned WDS-SEM and LA-ICPMS were used for phosphorus analysis while SIMS was used for oxygen analysis. A total of 5 shots were made close to the center of the metal drop. Each shot milled roughly $10 \mu \mathrm{m}$ in depth with a spot size of $30 \mu \mathrm{m}$. A sixth oxygen measurement was performed using SIMS to generate a depth profile of the oxygen concentration. This profile was obtained by milling from the center of the cross section of the droplet towards the interface of the droplet. This profile is shown in Figure 3. The summary of chemical analysis carried out by each technique is shown in Table VI.

The results mapped against the previously reported surface area profiles of the samples $(\mathrm{Ai} / \mathrm{A} 0)$ show that phosphorus had been removed from the droplet before 10 seconds of reaction time (see Figure 4). This was much sooner than the first visualization of emulsification in system 1, at 30 seconds. This leads to the conclusion that phosphorus exchange across the interface is not the reason for emulsification to occur, and the equilibration time for phosphorus between metal and slag is much shorter than the ideal predicted time of 120 seconds calculated previously. ${ }^{[1]}$

SIMS results show a large increase in oxygen concentration within the droplet at 20 seconds, as compared to the initial alloy (whose oxygen analysis was outsourced to $\mathrm{LECO}$ ). The $\mathrm{Fe}-\mathrm{FeO}$ equilibrium based on the

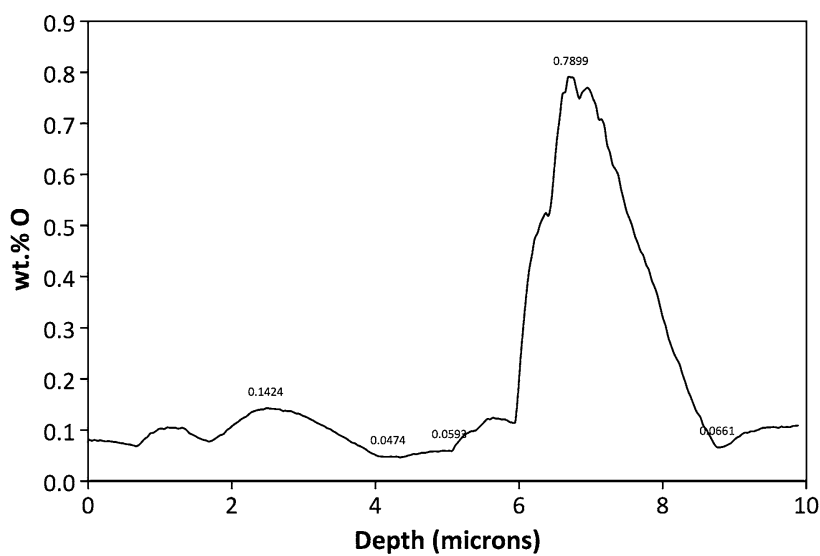

Fig. 3-Measured oxygen concentration as a function of depth into the 20-s sample (using a cross section) from Assis et al.

activity of $\mathrm{FeO}$ in the master slag has been predicted using FactSage, giving oxygen equilibrium content within the metal of $\sim 1000 \mathrm{ppm}$. This coupled with the calculated reduction in oxygen early on due to reaction with phosphorus giving the predicted oxygen content as a function of time mapped in Figure 4. SIMS analysis showed an average oxygen content of $0.213 \pm 0.139 \mathrm{wt}$ pct, significantly higher than the predicted equilibrium. Figure 3 also shows the heterogeneous distribution of oxygen radially through the droplet, with values from below equilibrium to those much higher. SIMS does not offer quantified data, however should be within a factor of 2 when appropriate standards are used.

From the points mentioned above, it is hypothesized that the exchange of oxygen across the metal/slag interface is the cause of emulsification. It is not known what would cause the mass oversaturation of oxygen within the droplet, however the mass transfer of oxygen back into the slag to reach equilibrium between 20 and 30 seconds, would be rapid. This along with the known fact that oxygen is a surfactant in a metal/slag system, leads the authors to the presented hypothesis.

In addition to the material presented, it was found that the cross-sectioned 0 -second sample contained a dispersion of particles throughout the droplet (see Figure 5). Local WDS of these particles showed them to be significantly higher in phosphorus than the bulk material (from 1.7 to $3.1 \mathrm{wt}$ pct P). These particles were not seen in the cross-sectioned images of later samples, where the phosphorus levels were seen to be drastically reduced (10 seconds onwards). Several images of the particles were used to calculate an area fraction of 0.4 pct; with this and the average particle concentration the amount of phosphorus removed is still significantly greater. It is possible that the phosphorus was held in a phase which was immiscible or did not melt at the same stage as the main bulk droplet; if this was the case, the high phosphorus phase may have been removed on the macro scale through particle migration from the bulk liquid to the droplet surface. Thus mechanical features rather than chemical reaction would be responsible for phosphorus removal. However, the challenges associated with the chemical analysis of oxygen and 
Table VI. Summary of Chemical Analysis Performed Using the Various Techniques Described

\begin{tabular}{|c|c|c|c|c|c|}
\hline \multirow[b]{2}{*}{ Reaction Time (s) } & \multicolumn{3}{|c|}{ (Wt Pct P) } & \multicolumn{2}{|c|}{ (Wt Pct O) } \\
\hline & ICP-MS & WDS-SEM & LA-ICPMS & LECO & SIMS \\
\hline Original alloy & 0.201 & $0.170 \pm 0.032$ & & 0.0737 & \\
\hline 0 & & $0.179 \pm 0.015$ & & & \\
\hline 10 & & $0.002 \pm 0.002$ & & & \\
\hline 20 & & $0.008 \pm 0.005$ & $0.0019 \pm 0.0001$ & & $0.213 \pm 0.139$ \\
\hline 30 & & $0.003 \pm 0.002$ & & & \\
\hline
\end{tabular}

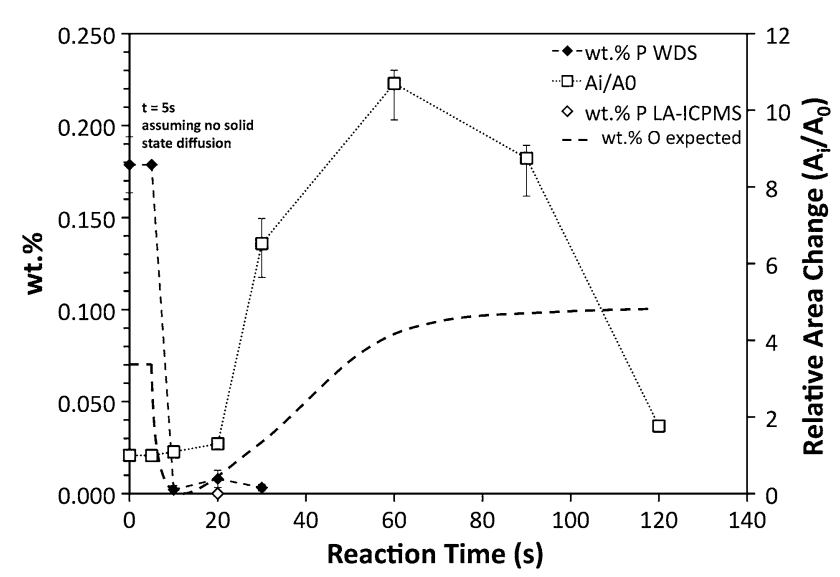

Fig. 4 -Expected oxygen concentration profile as a function of reaction time (LA-ICPMS only available at $20 \mathrm{~s}$ ).

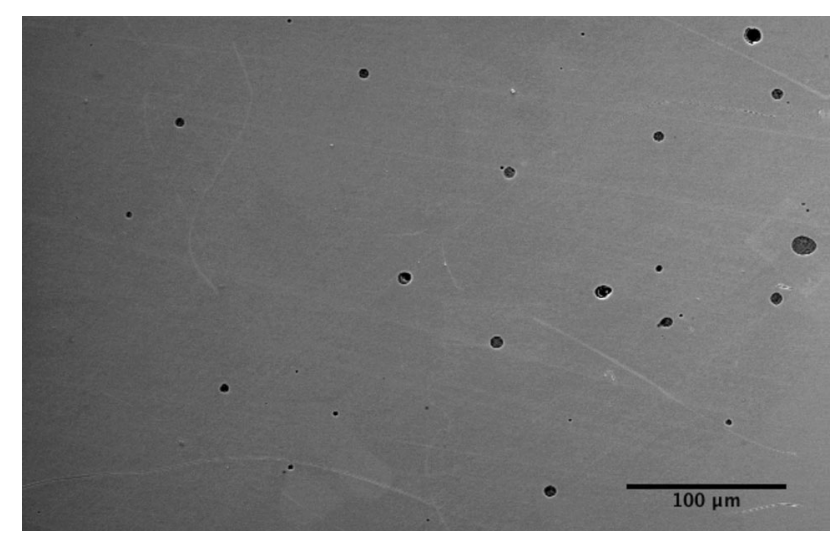

Fig. 5-Low magnification SEM picture of 'P-rich' particles.

phosphorous need to be overcome before we can confirm whether the two reactions indeed can be separated. The authors intend to interrogate this further in order to define a realistic mechanism for phosphorus removal.

\section{System 2, Dynamic Changes in Surface Area}

The expected reaction in system 2 is the electron exchange between ionic $\mathrm{Fe}$ and $\mathrm{O}$ in the slag to elemental $\mathrm{Fe}$ and $\mathrm{O}$ at the interface with the metal droplet:

$$
\left(\mathrm{Fe}^{2+}\right)+\left[\mathrm{O}^{2-}\right] \rightarrow[\mathrm{Fe}]+[\mathrm{O}]
$$

Again where material in () is in the slag phase, and material in [] is in the metal droplet.

3D reconstructions of XCT scans performed on system 2 quenched samples are shown in Figure 6. The initial sample of 10 seconds was selected as the starting point as this was when samples were shown to be fully liquid from system $1^{[1]}$ and used the same sample setup and user as previously, other sample times were selected in order to try and capture comparative emulsification data as to the initial study.

The surface area and volume of the droplets in system 2 samples were determined as described previously, ${ }^{[1]}$ the results of which are shown in Tables VII and VIII, respectively. The min, max, and selected surface areas/ volumes are based on manual selection of gray values.

The 10-second sample has melted and acquired a spherical shape, and emulsification takes place between 60 and 90 seconds. From Table VII the surface area of the drop stays relatively constant through the first 3 samples 6 to $8 \mathrm{~mm}^{2}$, followed by an increase by an order of magnitude to $65.75 \mathrm{~mm} .^{[2]}$ This increase in area is very similar to that seen in the previous study ${ }^{[1]}$; however there is a delay of $\sim 30$ seconds. This is clearly seen in the normalized area curves displayed in Figure 7. In system 2, oxygen is transferred from the slag into the metal, and the result appears to validate the hypothesis of oxygen transfer across the interface being responsible for the spontaneous emulsification (the behavior should occur regardless of oxygen transfer direction). The delay of 30 seconds, may be a product of the fact that no phosphorus exchange occurs in comparison to system 1 , however it may also be due to aspects such as inconsistent heat transfer inside the HT-CSLM, or other parameters that affected the differences seen in system 2 .

\section{System 3, Validation of Chemical Exchange Causing Spontaneous Emulsification}

A system designed with near equilibrium elemental distribution between slag and metal phases has been used to validate the hypothesis of chemical exchange across the interface being the cause of spontaneous emulsification.

System 3, notably has very low oxygen presence in the droplet (34 ppm) and zero $\mathrm{FeO} / \mathrm{SiO}_{2}$ in the slag phase (oxidation of $\mathrm{Fe}$ is thermodynamically unfavorable compared to all slag components). 

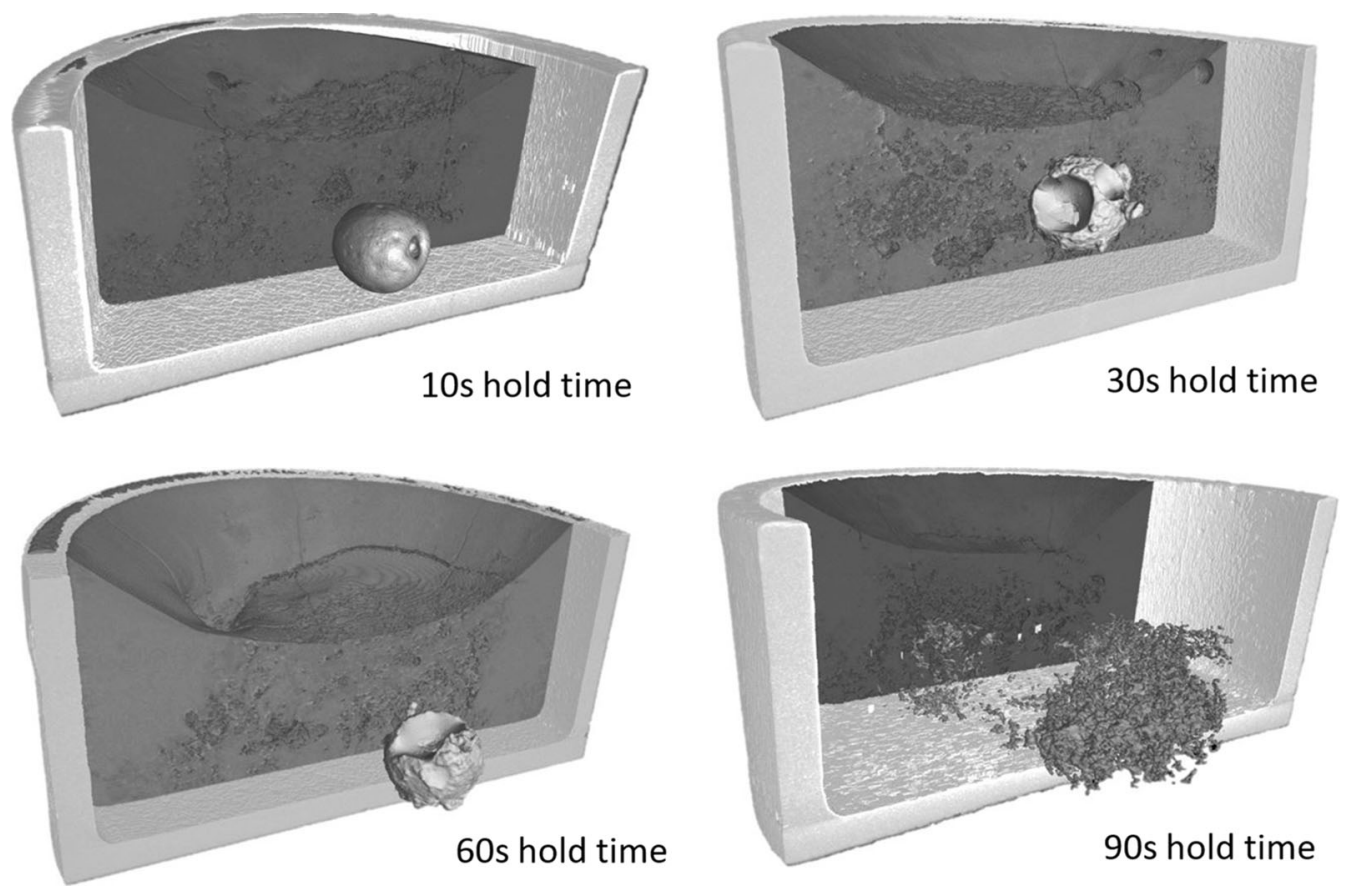

Fig. 6-Metal droplet 3D spatial geometry for different holding times in system 2.

Table VII. Raw Data for Surface Area Measurements of System 3

\begin{tabular}{|c|c|c|c|c|}
\hline Time (s) & Surface Area Selected $\left(\mathrm{mm}^{2}\right)$ & Surface Area Max $\left(\mathrm{mm}^{2}\right)$ & Surface Area Min $\left(\mathrm{mm}^{2}\right)$ & $\mathrm{Ai} / \mathrm{A} 0$ \\
\hline 10 & 6.60 & 6.60 & N.A & 1.00 \\
\hline 30 & 8.37 & 8.38 & 8.36 & 1.27 \\
\hline 60 & 6.84 & 6.86 & 6.83 & 1.04 \\
\hline 90 & 65.75 & 73.69 & 58.13 & 9.96 \\
\hline
\end{tabular}

Table VIII. Raw Data for Volume Measurements of System 2

\begin{tabular}{lccc}
\hline Time $(\mathrm{s})$ & Volume Selected $\left(\mathrm{mm}^{3}\right)$ & Volume Max $\left(\mathrm{mm}^{3}\right)$ & Volume $\mathrm{Min}\left(\mathrm{mm}^{3}\right)$ \\
\hline 10 & 1.29 & 1.30 & 1.28 \\
30 & 1.31 & 1.32 & 1.30 \\
60 & 1.19 & 1.19 & 1.18 \\
90 & 0.96 & 1.10 & 0.83 \\
\hline
\end{tabular}

It is known that thermal gradients across a medium can be a cause for spontaneous emulsification, ${ }^{[41]}$ and with the hot spot of the CSLM being $\sim 2 \mathrm{~mm}$ in diameter (smaller than the sample), and heat transfer being required from sample surface through the sample, it is unlikely the entire sample is constantly at the same temperature, especially during heating and during the early period of the programed isotherm.

System 3 being transparent in nature is allowed for the in situ viewing of the droplet throughout a reaction profile. It was seen that the droplet appears to stay spherical in shape throughout the period of viewing, well past the previously reported emulsification times.
Figure 8 also shows an XCT cross section of the entire sample, where the droplet is shown to be completely spherical and no Fe material was detected separate from the main mass of iron. This sample had a smoother surface than all previously reported samples not emulsified. The authors feel confident as a result that thermal gradients are not the cause of emulsification in this case.

\section{E. Mechanisms of Oxygen Transfer into the Droplet}

Due to the highly basic nature of the slags used in this study, it is likely a bi-layer would form at the interface with a cation and anion layering. ${ }^{[42]}$ With this in mind, it 


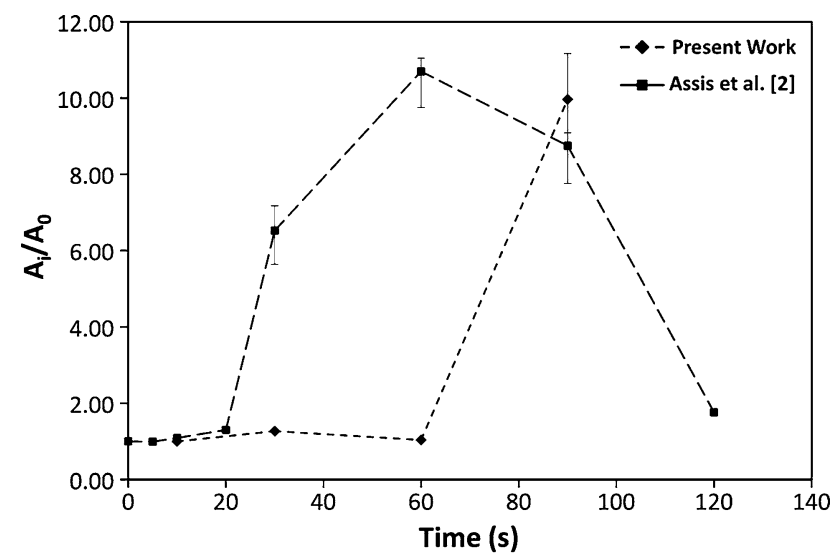

Fig. 7-Normalized surface area of metal droplets as a function of time, for system 2 where oxygen from the slag in entering the droplet, and system 1 where phosphorus from the droplet is being refined to the slag.

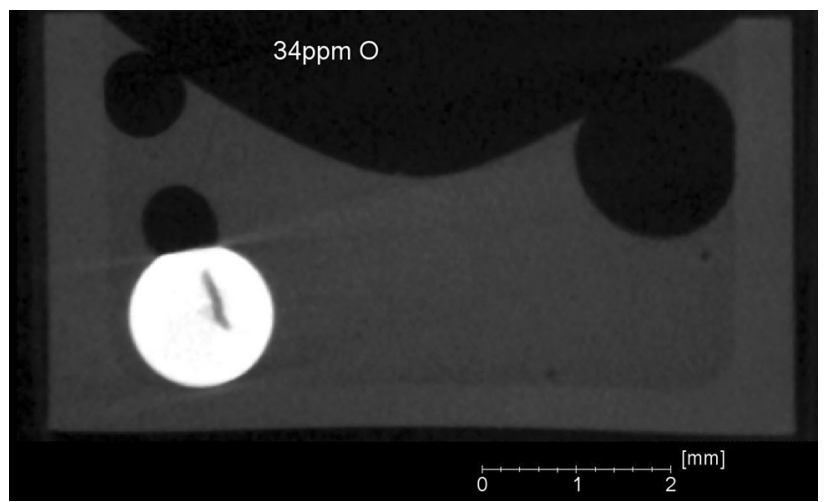

Fig. 8 - Ortho slice of CT scan of system 4 sample quenched at $90 \mathrm{~s}$.

is possible that a stabilizing interaction forms as the ions order into a favorable geometry lowering interfacial tension through charge matching with electrons in the liquid metal (electro capillary formation). Although this can reduce the energy required for phase mixing in the systems, the observation of system 3 where a basic slag is also used gives evidence that electro capillary action is insufficient to allow spontaneous emulsification alone.

If the work of Sanfeld ${ }^{[43]}$ is considered, stabilization of a phase or reaction in the bulk can be reverted due to either catalytic or reduced freedom (pinning of species) contributions through ordering at the interface of immiscible fluids. Hence it is proposed that the micro environment created by a bi-layer may allow for the disproportionation between ionic iron and oxygen at the interface, creating neutral species and allowing absorption into the non-charged liquid metal phase (as depicted for system 2), as opposed to the existence of stable $\mathrm{O}^{2-}$ and $\mathrm{Fe}^{2+}$ in the bulk metal oxide slurry. This process would still be governed by the mass transfer of material in the slag phase, however once ions enter the bi-layer the stabilization for disproportionation would still be present, and thus over the formation of atomic $\mathrm{O}$ is possible with regards to equilibrium concentration between the two phases.
It should be noted however, that the formation of atomic species and transport through the interface would disrupt the ordering of any formed bi-layer and hence expectantly reduce the stabilizing effects it forms. Thus this destabilization should be accounted for when considering the driving force during active chemical exchange across the interface; an addition to the energy barrier for chemical exchange previously discussed.

A further mechanism is the use of the droplet as an electron carrier. ${ }^{[4]}$ If we account for mass transport of $\mathrm{Fe}^{2+}$ in the slag to be the rate-limiting step, a depletion of this ion at the surface is likely. As a result the reaction mechanism may take advantage of the metal droplet reach through the slag (through not only its large size compared to that of chemical interaction, but also the continued growth of perturbations penetrating new areas of bulk slag) and the fast transport of electrons through the liquid metal to reduce the kinetic constraints on oxygen uptake. The steps for this mechanism are as follows:

1. Anionic oxygen transport across the interface from slag into the droplet

2. Transport of electrons through the metal droplet to a site in contact with an iron cation

3. Transfer of the electrons to the iron cation to form an iron atom

4. Uptake of the neutral iron atom into the droplet

These steps can be expressed by the redox reactions:

$$
\begin{gathered}
\left(\mathrm{O}^{2-}\right) \rightarrow[\mathrm{O}]+\left[2 e^{-}\right], \\
\left(\mathrm{Fe}^{2+}\right)+\left[2 e^{-}\right] \rightarrow[\mathrm{Fe}],
\end{gathered}
$$

where species in () are in the slag phase, and those in [] are in the metal phase. Where $\mathrm{O}^{2-}$ may be representative of a single oxygen ion or any reacting complex containing the ion.

This mechanism requires the movement of a charged oxygen ion into the non-charged metal droplet; this has a reliably large energetic cost and thus the concentration gradient is required to be large in order for this to happen at any meaningful rate. As such, this process may have a large influence early on in the experiment, however as the process continues it is unlikely that this mechanism could be responsible for the over saturation of oxygen seen from the SIMS results.

To interrogate these effects further, the authors propose experimentation with lower basicity slags, and electrochemical experimentation. By these methods it may be possible to isolate each effect and give quantification to its participation in the phenomena reported here.

\section{CONCLUSIONS AND FURTHER WORK}

Chemical analysis of samples from a previous study by Assis et al. ${ }^{[1]}$ appear to show that phosphorus movement across the interface between a metal drop and slag is not responsible for spontaneous emulsification. Instead it is hypothesized that oxygen partition in 
either direction across the interface causes a drop in interfacial tension through surfactant effects and active refining. This is supported by a system where only oxygen content was drastically away from equilibrium, showing similar behavior to that seen previously (apart from a slight delay).

A replication of the previous study has also been carried out by another user with a different HT-CSLM. This showed very different melting times for the metal droplet and as a result a changed pathway of droplet morphology through the reaction period. Discussion has been given as to why these differences may have been seen, with heating performance, and sample preparation being the clearest possibilities. It has also been seen that prior to emulsification the droplet appears to show substantial roughening over the entire surface of the droplet, a link to the emulsification mechanism.

The authors intend to continue this work, with the use of optically transparent slags, and Fe-Al alloys, in an attempt to visualize the emulsification in situ using the HT-CSLM. The study will also be extended to encompass the effects of other common impurities seen in steel makings, such as carbon and sulfur.

\section{OPEN ACCESS}

This article is distributed under the terms of the Creative Commons Attribution 4.0 International License (http://creativecommons.org/licenses/by/4.0/), which permits unrestricted use, distribution, and reproduction in any medium, provided you give appropriate credit to the original author(s) and the source, provide a link to the Creative Commons license, and indicate if changes were made.

\section{REFERENCES}

1. A.N. Assis, J. Warnett, S. Spooner, R.J. Fruehan, M.A. Williams, and S. Sridhar: Metall. Mater. Trans B, 2015, vol. 46B, pp. 568-76.

2. J. Gustavsson: Reactions in the Lower Part of the Blast Furnace with Focus on Silicon, Royal Institute of Technology, Stockholm, 2004.

3. A. Yang: A Pre-study of Hot Metal Desulphurization, Royal Institute of Technology, Stockholm, 2012.

4. S. Basu: Studies on Dephosphorisation During Steelmaking, Royal Institute of Technology, Stockholm, 2007.

5. M.A. Tayeb, R.J. Fruehan, and S. Sridhar: International Symposium on Liquid Metal Processing \& Casting, 2013, pp. 353-58.

6. M.A. Tayeb, R.J. Fruehan, and S. Sridhar: AISTech., 2014, pp. 1.

7. N. Sano and P.V. Riboud: Advanced Physical Chemistry for Process Metallurgy, Academic Press, San Diego, 1997.

8. C.P. Manning and R.J. Fruehan: Metall. Mater. Trans. B, 2013, vol. 44B, pp. 37-44.

9. P. Wei, M. Sano, M. Hirasawa, and K. Mori: ISIJ Int., 1993, vol. 33, pp. 479-87.

10. P. Wei, M. Sano, and M. Hirasawa: ISIJ Int., 1991, vol. 31, pp. 358-65.

11. S. Kitamura, S. Saito, K. Utagawa, H. Shibata, and D.G.C. Robertson: ISIJ Int., 2009, vol. 49, pp. 1838-44.

12. J. Diao, X. Liu, T. Zhang, and B. Xie: Int. J. Miner. Metall. Mater., 2015, vol. 22, pp. 249-53.

13. L. Muhmood, N.N. Viswanathan, and S. Seetharaman: Metall. Mater. Trans. B, 2011, vol. 42B, pp. 460-70.

14. M. Martín, M. Rendueles, and M. Díaz: Chem. Eng. Res. Des., 2005, vol. 83, pp. 1076-84.
15. Y. Chung and A. Cramb: Metall. Mater. Trans. B, 2000, vol. 31B, pp. $957-71$.

16. H. Gaye and P.V. Riboud: Metall. Trans. B, 1977, vol. 8B, pp. 409-15.

17. A. Jakobsson, M. Nasu, J. Mangwiru, K.C. Mills, and S. Seetharaman: Philos. Trans. R. Soc. A Math. Phys. Eng. Sci., 1998, vol. 356, pp. 995-1001.

18. M. Rhamdhani, G. Brooks, and K. Coley: Metall. Mater. Trans. B, 2006, vol. 37B, pp. 1087-91.

19. M. Rhamdhani, K. Coley, and G. Brooks: Metall. Mater. Trans. B, 2005, vol. 36B, pp. 591-604.

20. M. Rhamdhani, K. Coley, and G. Brooks: Metall. Mater. Trans. B, 2005, vol. 36B, pp. 219-27.

21. C. Cicutti, M. Valdez, T. Perez, J. Petroni, A. Gomez, R. Donayo, and L. Ferro: 12 IAS Steelmaking Seminar, 1999, p. 250.

22. S. Sabah and G. Brooks: Metall. Mater. Trans. B, 2014, vol. 46B, pp. 863-72.

23. S. Sabah and G. Brooks: ISIJ Int., 2014, vol. 54, pp. 836-44.

24. D.J. Price: Process Engineering of Pyrometallurgy, 1974, p. 8-15.

25. Q. He and N. Standish: ISIJ Int., 1990, vol. 30, pp. 356-61.

26. N. Dogan, G.A. Brooks, and M.A. Rhamdhani: ISIJ Int., 2011, vol. 51, pp. 1086-92.

27. N. Dogan and G.A. Brooks: ISIJ Int., 2011, vol. 51, pp. 10931101.

28. P. Kozakevitch: J. Miner. Met. Mater. Soc., 1969, vol. 22, pp. 57-58.

29. G. Brooks, Y. Pan, and K. Coley: Metall. Mater. Trans. B, 2005, vol. 36B, pp. 525-35.

30. J. Schoop, W. Resch, and G. Mahn: Ironmak. Steelmak., 1978, vol. 2, pp. 72-79.

31. M.S. Millman, A. Overbosch, A. Kapilashrami, D. Malmberg, and M. Brämming: Ironmak. Steelmak., 2013, vol. 40, pp. 460-69.

32. M.S. Millman, A. Overbosch, A. Kapilashrami, D. Malmberg, and M. Brämming: Trans. Indian Inst. Met., 2013, vol. 66, pp. 525-34.

33. D. Min and R.J. Fruehan: Metall. Mater. Trans. B, 1992, vol. 23B, pp. 29-37.

34. C. Molloseau and R.J. Fruehan: Metall. Mater. Trans. B, 2002, vol. 33 B, pp. $335-44$.

35. T. Gare and G.S.F. Hazeldan: Ironmak. Steelmak., 1981, vol. 8, pp. $169-81$

36. H. Yin, H. Shibata, T. Emi, and M. Suzuki: ISIJ Int., 1997, vol. 37 , pp. $936-45$.

37. H. Yin, H. Shibata, T. Emi, and M. Suzuki: ISIJ Int., 1997, vol. 37, pp. 946-55.

38. H. Shibata, Y. Arai, M. Suzuki, and T. Emi: Metall. Mater. Trans. $B$, 2000, vol. 31B, pp. 981-91.

39. H. Shibata, T. Emi, H. Yin, M. Suzuki: 4th Decennial International Conference on Solidification Processing, 1997, vol. 37, pp. 230-33.

40. J Kumar, A Attridge, PKC Wood, and MA Williams: Measur. Sci. Tech., 2011, vol. 22, p. 035105.

41. J.C. López-Montilla, P.E. Herrera-Morales, S. Pandey, and D.O. Shah: J. Dispers. Sci. Technol., 2002, vol. 23 (1-3), pp. 219-68.

42. B.V. Patrov: Surface Phenomena in Metallurgical Processes, 1st ed., Springer, Berlin, 2012, pp. 129-133.

43. A. Sanfeld: Philos. Trans. R. Soc. A Math. Phys. Eng. Sci., 1998, vol. 356 , pp. 819-28.

44. W. Freyland: Colombic Fluids, 1st ed., Springer, Berlin, 2011, pp. 5-44.

45. M.S. Millman, A. Kapilashrami, M. Bramming, and D. Malmberg: Imphos: Improving Phosphorus Refining, Publications Office of the European Commision, Brussels, 2011.

46. W. Resch: Kinetics of $P$ Removal During O Top-Blowing Processes for P-Rich Fe, Technical University, Clausthal, 1976.

47. O.K. Tokovoi, A.I. Stroganov, and D.Y. Povolotskii: Steel USSR, 1972, vol. 2, pp. 116-17.

48. S.C. Koria and K.W. Lange: Ironmak. Steelmak., 1986, vol. 13, pp. 236- 40.

49. V.I. Baptizmanskii, V.B. Okhoskii, K.S. Provirin, G.A. Shcherdrin, Yu.A. Ardelyan, and A.G. Velichko: Steel USSR, 1977, vol. 7, pp. 329-31

50. HW Meyer, WF Porter, GC Smith, and J Szekely: J. Met., 1968, vol. 20 , pp. $35-42$

51. R.F. Block, A. Masui, and G. Stolzenberg: Arch. Eisenhuttenwes, 1973, vol. 44, pp. 357-61

52. R.C. Urquhart and W.G. Davenport: Can. Metall. Q., 1973, vol. 12 , pp. 507-16. 